\title{
Original
}

\section{Nutrición enteral temprana, en pacientes con abdomen abierto}

\author{
Mario Sánchez-Arias
}

\section{Resumen}

Objetivo: Observar la factibilidad de la nutrición enteral en el paciente crítico quirúrgico con abdomen abierto y valorar su avance y posibles complicaciones.

Método: Estudio observacional descriptivo de los pacientes que ingresaron a la Unidad de Cuidado Intermedio Quirúrgico de la Sección de Cirugía del Hospital Dr. R.A. Calderón Guardia, con abdomen abierto, de junio de 2003 a noviembre de 2004, y en quienes se colocó una sonda de nutrición en yeyuno.

Resultados: Se observaron 15 pacientes, con una edad promedio de 46 años, con abdomen abierto y acceso nasoyeyunal el $80 \%$ y yeyunal directo el $20 \%$, a los cuales se les inició nutrición enteral temprana ( $36 \mathrm{hrs}$ ) con una fórmula polimérica, a razón de $23 \mathrm{cc} / \mathrm{hr}$, que se avanzó a razón de 6.6cc/día, por un promedio de 9 días, alcanzando un $80 \%$ de ellos el $80 \%$ de sus requerimientos estimados, en 4.6, días. No hubo mortalidad en la serie, aunque sí intolerancia al régimen $(33.3 \%)$, principalmente por persistencia de fístula; tales pacientes se pasaron a nutrición parenteral.

Conclusiones: La nutrición enteral puede usarse en pacientes con abdomen abierto, si se les procura un acceso yeyunal y se les proporciona en volúmenes y aumentos discretos, de acuerdo con la condición del intestino.

Descriptores: Nutrición enteral temprana en paciente crítico, nutrición enteral en paciente con abdomen abierto, nutrición enteral.

Keywords: Early enteral nutrition, open abdomen, nutrition in critical care.

Recibido: 26 de agosto de 2005

Aceptado: 8 de noviembre de 2005

Sección de Cirugía, Hospital Dr. R.A. Calderón G., Unidad de soporte nutricional.

Abreviaturas: $\mathrm{AbAb}$, abdomen abierto; A.B, arma blanca; CCSS, Caja Costarricense de Seguro Social; Hb, hemoglobina; Kcal, kilocalorías; Sepsis Ab, sepsis abdominal; UCl, Unidad Cuidado Intermedio.

Correspondencia: Email: masanchezarias@yahoo.com masanche@racsa.co.cr

ISSN 0001-6002/2006/48/1/12-16 Acta Médica Costarricense, ○2006 Colegio de Médicos y Cirujanos
A pesar de que se ha acumulado mucha evidencia en la bibliografía, del beneficio en todo nivel que tiene el paciente al que se nutre enteralmente en forma temprana ${ }^{1}$, ha sido lento a través de las últimas cuatro décadas, hacer que los cirujanos valoren la importancia del soporte nutricional en sus pacientes. Pese a esto cada vez es más frecuente ver cómo los colegas se hacen la pregunta de oro: ¿“y cómo voy a nutrir este paciente"? ${ }^{2}$. Afortunadamente, los grupos de soporte nutricional, con el rápido avance de la nutrición parenteral en los años 70 y $80^{3,4} \mathrm{y}$ con el vertiginoso desarrollo de la nutrición enteral en los años 90 y $2000^{5,6}$, siempre tienen una respuesta a esa inquietud y estamos en capacidad de proporcionar el esquema necesario, por vía oral, enteral o parenteral, con los múltiples accesos de que disponemos ${ }^{7-9}$.

Para el cirujano era prohibitivo dar nutrición enteral después de cirugía abdominal mayor, si el paciente no cumplía con los criterios de: peristalsis presente auscultable, canalización de gases y haber tenido la primera deposición ${ }^{10}$. Con los estudios de bioelectromotricidad intestinal se comprobó que el ileo posoperatorio era predominantemente colónico (4 días), luego gástrico (dos días) y por último de intestino delgado (12 hrs); más aún, que el estímulo 
del nutriente en la luz yeyunal en forma temprana favorecía mayormente la resolución del ileo intestinal ${ }^{11-14}$.

Con este conocimiento se han llevado a cabo múltiples trabajos usando acceso nasoyeyunal, gastroyeyunal o yeyunal directo, en pacientes de cirugía abdominal mayor ${ }^{15-18}$. Debido al éxito en el manejo de estos pacientes, a su más rápida recuperación, menor índice de complicaciones y menores estancias hospitalarias, se ha estandarizado la nutrición posoperatoria inmediata ${ }^{19,20}$.

Alimentándose de esta experiencia se han empezado a nutrir enteralmente pacientes que en el pasado no eran candidatos para ello por vía enteral (de cuidado intensivo, con ventilación mecánica, con vasopresores, trauma, etc.) ${ }^{21,22}$. En esta comunicación nos ocuparemos de los pacientes con condiciones agudas, en quienes debe dejarse el abdomen abierto $^{23}$, para su manejo apropiado, llevando prospectivamente un protocolo observacional para dar soporte nutricional enteral (en forma temprana) ${ }^{24}$ al menos por 4 días, a pacientes que requirieron quedar con bolsa de "Bogotá" con el fin de valorar su tolerabilidad, posibilidades de infusión, kilocalorías y proteínas administradas, tiempo de duración y complicaciones, entre otros ${ }^{27}$.

\section{Materiales y métodos}

Se tomaron los pacientes admitidos a la Unidad de Cuidados Intermedios Quirúrgicos del Hospital Dr. R. A. Calderón Guardia, de la CCSS, después de cirugía abdominal mayor y que ameritaron quedar con abdomen abierto, cubierto con bolsa plástica ("Bogotá"), de junio de 2003 a noviembre de 2004, y quienes salieron con acceso enteral yeyunal confirmado en el momento de la cirugía (sonda nasoyeyunal $\mathrm{N}^{\circ} 12$ de poliuretano o sonda $\mathrm{N}^{\circ} 16$ de yeyunostomía), para valorar la nutrición enteral a tolerancia; se incluyeron en el estudio los que duraron al menos 4 días con abdomen abierto y nutrición enteral, con fórmula polimérica (Ensure $\AA)$.

Se estudia el tiempo de inicio del uso del acceso enteral, la velocidad de infusión al comienzo, el avance por día, la duración del soporte enteral, el promedio de kcal y proteínas administradas el día último previo al cierre del

\begin{tabular}{|c|c|c|}
\hline \multicolumn{3}{|c|}{$\begin{array}{l}\text { Cuadro 1. Estado nutricional, de pacientes } \\
\text { con Abdomen abierto y nutrición enteral, } \\
\text { Según valoración global subjetiva }\end{array}$} \\
\hline Categoría & Valor inicial & Valor final \\
\hline Bien nutrido & $13.2 \%$ & $0 \%$ \\
\hline Riesgo desnutrición & $33 \%$ & $40 \%$ \\
\hline Desnutrido & $46.2 \%$ & $60 \%$ \\
\hline Obeso & $6-6 \%$ & $0 \%$ \\
\hline
\end{tabular}

abdomen, si se alcanzó o no el 80\% del requerimiento con su duración, así como las complicaciones desarrolladas y la necesidad de cambio a nutrición parenteral. Los datos colectados corresponden al período en que el paciente cursó con el abdomen abierto.

\section{Resultados}

Se estudiaron un total de 15 pacientes, con un promedio de edad de 46 años, con una distribución por sexo de $\mathrm{H}$ : 53\% y M: $47 \%$. El cambio en el estado nutricional antes y después del período de nutrición enteral se muestra en el Cuadro 1. La albúmina promedio de los pacientes al inicio fue de $1.9 \mathrm{y}$ al final de $1.8 \mathrm{~g} / \mathrm{dl}$, la hemoglobina de $9.11 \mathrm{y}$ 9.3 respectivamente, leucograma 14.750 y 13210 con linfocitos de menos 1.500 en el $50 \%$ de los casos inicial y $40 \%$ pos tratamiento. Un total de $60 \%$ de pacientes con anastomosis (pre sitio de NE 33\%, pos 27\%). Se usó un acceso nasoyeyunal en $80 \%$, y yeyunostomía en $20 \%$. El tiempo de inicio de uso después de colocada la sonda fue en promedio de $39 \mathrm{hrs}$, con un volumen de 23 cc por hora, dándose el tratamiento por un promedio de 9 días con fórmula polimérica, aumentando en la infusión a razón de $6.6 \mathrm{cc} /$ día. El 80\% de los pacientes alcanzaron el $80 \%$ de los requerimientos calculados con regla simple de $30 \mathrm{kcal} / \mathrm{kg}$, en un promedio de 4.6 días. Se les administró un promedio de $1616 \mathrm{kcal}$ al final de cierre del abdomen con un aporte proteico de $52.17 \mathrm{~g}$. En relación a la tolerabilidad un $53.3 \%$ del grupo se cambio a la vía oral corriente una vez que se cerró el abdomen, un $13.3 \%$ siguió con nutrición enteral pos cierre de la pared y un 33.3\% no toleró el régimen y fue necesario cambiarlo a nutrición parenteral por diferentes motivos. Los diagnósticos, la evolución, las operaciones practicadas y las complicaciones se detallan en el Cuadro 2. Todos los pacientes sobrevivieron al momento del cierre del abdomen (100\%).

\section{Discusión}

Ciertamente la nutrición no es una terapéutica de emergencia, que deba usarse antes o simultáneamente con la reanimación de un estado de choque, sin embargo conforme se ha avanzado en el conocimiento de la fisiopatología del paciente agudamente enfermo ${ }^{28,29}$, se ha reconocido que el soporte nutricional es una de las acciones que más sostienen al paciente ${ }^{30}$ y pueden evitar hasta su curso a un síndrome de falla multisistémica ${ }^{31-33}$.

De modo que hoy en día no solo juega un papel muy importante en cuanto a disminuir (ya que es imposible evitar, una vez se dispara la cascada de consumo ${ }^{34}$, el catabolismo a que se ve sometido el enfermo que ha tenido una 


\begin{tabular}{|c|c|c|c|c|}
\hline \multicolumn{5}{|c|}{$\begin{array}{c}\text { Cuadro 2. Pacientes con abdomen abierto y nutrición } \\
\text { enteral (operaciones y complicaciones). }\end{array}$} \\
\hline Diagnósticos & Evol & Operaciones & Complicaciones & $\begin{array}{l}\text { Cambio } \\
\text { NP }\end{array}$ \\
\hline Ca Gástrico,Sepsis Ab. & salón & $\begin{array}{l}\text { Gastrect.Total+YRoux } \\
\text { Rafias,gastroyey.AbAb }\end{array}$ & no & no \\
\hline $\begin{array}{l}\text { Hernia hiatal recidivante. } \\
\text { Rupt gástrica.Sepsis } A b .\end{array}$ & $\mathrm{UCl}$ & $\begin{array}{l}\text { Re-Nissen, Rafias } \\
\text { gástri-Cas, AbAb. }\end{array}$ & Fístula persistente & si \\
\hline $\begin{array}{l}\text { Ruptura gástrica. } \\
\text { Fístula enterocutánea. Sepsis Ab. }\end{array}$ & salón & $\begin{array}{l}\text { Rafias gástricas múltipl } \\
\text { Lavados abdom. AbAb }\end{array}$ & Fístula persistente & si \\
\hline $\begin{array}{l}\text { Perforación divertículo de } \\
\text { sigmoides. Sepsis Ab. }\end{array}$ & salón & $\begin{array}{l}\text { Resección, Operación } \\
\text { Hartman.Lavados AbAb }\end{array}$ & no & no \\
\hline $\begin{array}{l}\text { Trauma cerrado abominal } \\
\text { Rupt colédoco e hígado }\end{array}$ & salón & $\begin{array}{l}\text { Hepático-yeyuno,sutura } \\
\text { empaque hígado.AbAb }\end{array}$ & no & no \\
\hline $\begin{array}{l}\text { Perforación colon derech } \\
\text { Sepsis Ab. }\end{array}$ & salón & $\begin{array}{l}\text { Colectomía derecha, } \\
\text { Ileostomía, AbAb }\end{array}$ & no & no \\
\hline $\begin{array}{l}\text { Traum penet A-B,lesión } \\
\text { páncreas, fístula P,.Sepsis }\end{array}$ & salón & $\begin{array}{l}\text { Pancreatectomía.distal, } \\
\text { Pancreato yeyuno.AbAb }\end{array}$ & no & no \\
\hline $\begin{array}{l}\text { Pancreat crónica. Fístula } \\
\text { pancreática. Sepsis Ab }\end{array}$ & $\mathrm{UCl}$ & $\begin{array}{l}\text { Pancreatoyeyuno, Rafias } \\
\text { Lavados. AbAb }\end{array}$ & $\begin{array}{l}\text { Distensión, ileo, } \\
\text { Descomp. Hemodi- }\end{array}$ & si \\
\hline $\begin{array}{l}\text { Perforación divertículo de } \\
\text { sigmoides. Sepsis } A b\end{array}$ & salón & $\begin{array}{l}\text { Resección con anastom } \\
\text { Rafias, lavados. AbAb. }\end{array}$ & no & no \\
\hline $\begin{array}{l}\text { Perforación divertículo de } \\
\text { colon izq. Sepsis Ab }\end{array}$ & $\mathrm{UCl}$ & $\begin{array}{l}\text { Laparotomía, cecostomía } \\
\text { Lavados. AbAb }\end{array}$ & Fístula persistente & si \\
\hline $\begin{array}{l}\text { Traum penet x A-Fuego } \\
\text { Les:gást.bazo -páncr. SAb }\end{array}$ & $\mathrm{UCl}$ & $\begin{array}{l}\text { Gastrect, esplenectomía } \\
\text { Pancreatect-distal AbAb }\end{array}$ & Diarrea, regurgitación & si \\
\hline $\begin{array}{l}\text { Perforación sigmoides,Pte } \\
\text { Irradiado Sepsis Ab }\end{array}$ & salón & $\begin{array}{l}\text { Resección, Operación } \\
\text { Hartman.Lavados.AbAb }\end{array}$ & no & no \\
\hline $\begin{array}{l}\text { Perforación divertículo } \\
\text { Colon izq. Sepsis Ab }\end{array}$ & salón & $\begin{array}{l}\text { Resección, Operación } \\
\text { Hartman.Lavados.AbAb }\end{array}$ & no & no \\
\hline $\begin{array}{l}\text { Trauma penet.A-B.Lesión } \\
\text { Gástrica. Sepsis Ab. }\end{array}$ & salón & $\begin{array}{l}\text { Gastrectomía total } \\
\text { Rafias.Lavados.AbAb }\end{array}$ & no & no \\
\hline $\begin{array}{l}\text { Traum cerrados Ab.Lesión } \\
\text { Gástrica-colon. Sepsis Ab }\end{array}$ & salón & $\begin{array}{l}\text { Rafias. Lavados } \\
\text { AbAb }\end{array}$ & no & no \\
\hline
\end{tabular}

injuria importante. Es por eso que hoy se habla en términos más amplios como: "farmaconutrición" "35,36 (considerando la fórmula de soporte nutricional como un fármaco más) y se dispone inclusive de soluciones con elementos esenciales o condicionalmente esenciales que ayudan al sistema inmunológico a reaccionar mejor en ciertas nutrición o de desgaste a lo que llamamos "inmunonutrición"37-40. De igual forma se ha avanzado en dar nutrición enteral a pacientes que en décadas pasadas no eran ni siquiera considerados candidatos $^{41}$ y solo se les daba nutrición parenteral; entre ellos muy especialmente los pacientes con abdomen abierto, ya que se consideraba que el intestino expuesto cubierto con una malla o una bolsa plástica estaba paralizado y no se le podía dar nutrientes.
En este trabajo observacional hemos seguido 15 pacientes, con edad promedio 46 años, con igual distribución por sexo, con padecimientos muy serios, que ameritaron grandes cirugías y a quienes se les dejó el abdomen abierto para subsecuentes revisiones y lavados (Cuadro 1) por sepsis abdominal (93.4\%) no controlada (leucogramas 14750 al inicio). Si bien no se pretende alcanzar metabólicamente a pacientes tan severamente comprometidos y en tan corto tiempo, al menos se evita que se catabolicen muy rápidamente, lo que se ve en el porcentaje de pacientes que terminó estando en el grupo de riesgo de desnutrición y desnutrido, muy similar antes y después del período de manejo enteral. Tratamos pacientes con albúminas y hemoglobinas iniciales de muy bajas, lo que es ya de por sí un 
dato de morbilidad por desnutrición ${ }^{42}$, las cuáles no se modificaron por lo corto del período de observación pero también por el apoyo nutricional que recibieron.

El acceso yeyunal es muy importante para evitar regurgitación gástrica y broncoaspiración ${ }^{43}$, todo nuestro grupo fue nutrido directamente en el yeyuno $(80 \%$ se les colocó sonda nasoyeyunal en el momento que se decidió dejar abdomen abierto, 20\% ya tenían yeyunostomía cuando se dejó abdomen abierto) con la fórmula polimérica disponible en el momento en el $\mathrm{HCG}$ que fue el Ensure ${ }^{\circledR}$ de Abbot Laboratories, Chicago, Il, EUA.

Después de colocada la vía yeyunal, se inició el soporte tan pronto como fue posible $e^{44,45}$ lo que califica como nutrición temprana $(<48 \mathrm{hrs})$. Se inició con un volumen bajo y se fue viendo la tolerancia sin poner en riesgo el paciente, alcanzando un promedio de aumento menor al alcanzado en pacientes con nutrición enteral temprana en pacientes médicos ${ }^{46} \mathrm{o}$ pos-operatoria con abdomen cerrado ${ }^{47}$ (debido a las condiciones críticas que tienen estos intestinos de: edema, engrosamiento, obstrucción, acartonamiento, etc).

El tiempo de tratamiento fue de 9 días (mientras el paciente tuvo abdomen abierto), alcanzando un porcentaje significativo de ellos un $80 \%$ de sus requerimientos, en un tiempo relativamente corto, que es un tiempo muy razonable para enfermos con condiciones tan agudas. De tal manera que al término de la condición "abdomen abierto", los enfermos estaban recibiendo una buena cantidad de calorías, así como aporte proteico, suficientes para evitar su autodesgaste $^{48}$.

Debe tomarse en cuenta que un $60 \%$ de éstos pacientes tenían además algún tipo de anastomosis en el tubo digestivo proximal al sitio de alimentación y un $27 \%$ en un punto distal al yeyuno, lo que nos indica nuevamente que no es una contraindicación absoluta dar nutrición enteral temprana a pacientes con anastomosis recientes.

Como era de esperarse, la tolerancia con todos estos aspectos mencionados fue menor; sin embargo solo un tercio de nuestros enfermos $(33.3 \%)$ no toleraron el régimen y fue necesario pasarlos a nutrición parenteral, principalmente por persistencia de fístula y menos por otras complicaciones $^{49}$ como se ve en la Cuadro 1, quienes tuvieron que permanecer en la Unidad de Cuidado Intensivo más tiempo. El resto salieron al salón corriente una vez que se les resolvió la condición de abdomen abierto, volviendo a tomar vía oral normal en un $53.3 \%$ y solo un $13.3 \%$ fue necesario continuar con la nutrición enteral por la sonda por unos días más. Durante el período que se mantuvieron con abdomen abierto y nutrición enteral, no tuvimos mortalidad en esta serie, a pesar de que es conocido que esta condición por si sola tiene mortalidades que oscilan $25-40 \%{ }^{50}$.

El autor reconoce que la casuística es baja y que no es un estudio comparativo, sin embargo esta experiencia permite pensar que si es posible nutrir enteralmente (en el yeyuno) a los pacientes que quedan con el abdomen abierto, dando una cantidad calórica y proteica suficiente para disminuir su catabolismo y su evolución a falla orgánica múltiple $^{51}$. Se debe ser cauto en cuanto a los volúmenes de inicio y al avance diario así como con los pacientes que tienen anastomosis, con el fin de no someterlos a una sobrecarga. Finalmente debe tenerse siempre la posibilidad de cambiar a nutrición parenteral ${ }^{52}$ si hay intolerancia o darles nutrición mixta cuando el aporte enteral logrado no sea suficiente.

\section{Agradecimiento}

Al grupo interdisciplinario de soporte nutricional del Hospital Dr. R. A. Calderón Guardia. (Dr: J.I.Padilla, J.Ramírez, Lic.M. Arias, Lic. M.Fallas, Lic. C.Vargas).

\section{Abstract}

Ojective: To observe enteral nutrition feasibility in patients with "open abdomen" their evolution and complications.

Methods: This is an observational study of patients with "open abdomen" who were admitted to the Intermediate Care Unit of the Surgical Service at the Calderón Guardia Hospital between june 2003 to November 2004, with a yeyunal access for nutrition.

Results: Fifteen patients were studied with an average age of 36 years, all of them with open abdomen and jejunal access, who got early enteral nutrition ( $36 \mathrm{hrs}$ ) with a polymeric formula, starting at $23 \mathrm{cc} / \mathrm{hrs}$ and were then advanced $6.6 \mathrm{cc} /$ daly for 9 days, reaching $80 \%$ of the patients, $80 \%$ of threir requirements in 4.6 days. There was not mortality but $33 \%$ did not have good tolerance because of persistent leakage. Those were accordingly changed to total parenteral nutrition.

Conclusions: Enteral nutrition can be used in patients with open abdomen if they have a jejunal access and are fed with small volumes and are advanced slowly according to the function of their small bowel .

\section{Referencias}

1. Broga M., Gianotti L, Gentilini O. Early postoperative enteral nutrition improves gut oxygenation and reduce costs compared with TPN. Crit.Care.Med. 2001; 29:242-248.

2. Moore F, Moore E., Jones T, McCroskey BL, Peterson VM. TEN vrs TPN fallowing major abdominal trauma reduced septic. J Trauma 1989; 29:916-922.

3. Wagner DR, Elmore MF, Tate JT. Combined parenteral and enteral nutrition in severe trauma. Nutr Clin Practice 1992; 7:113-116. 
4. Keretz R.L, Lipman TO, Klein S. AGA technical review: parenteral nutrition. Gastroenterology 2001; 121:970-1001.

5. Frost P, Bihari D. The route of nutrition support in the critically ill. Nutrition 1997; 13 (Suppl): 585-635.

6. Lowry SF. The route of feeding influence injury responses. J Trauma 1990; 30:S10-S15.

7. ASPEN Board of directors: guidelines for the use of parenteral and enteral nutrition in adult and pediatric patients. JPEN 1993; 17:15A$52 \mathrm{~A}$.

8. Pendley C. Gekle R., Campbell S. Enteral Nutrition Support in critical care. 1994 Ross Products Division, Abbot laboratories.

9. Weimam A, Braunert M, Muller T, Bley T, Wiedemann. Feasibility and safety of needle catheter yeyunostomy for enteral nutrition. JPEN 2004; 28: 324-327.

10. Schmith H., Martindale R. The gastrointestinal tract in critical illness. Curr. Opin Clin. Nutr. Metab. Care. 2001; 4: 547-551.

11. Carlin C.B., Scanlon P.H., Wagner D.A.. Gastric empthying in trauma patients. Dig Surg 1999; 16:192-196.

12. Kao C.H., Changlai S.P., Chieng P.V. Gastric empthying in head trauma patients. Am J Gastroenteerol. 1998; 93:1108-1112.

13. Weekes E., Elia M. Observations on the patterns of 24 hour energy expediture changes in body composition and gastric empthying in head injured patients receiving nasogastric tube feeding. JPEN 1996; 20: 31-37.

14. Lewis S.J., Egger M., Sylvester P., Thomas S. Early enteral feeding versus "nil by mouth" after GI surgery. BMJ 2001; 323:773.

15. Boulanger B.R., Brennemann F.D., Rizoli S.B. Insertion of a transpyloric feeding tube during laparotomy in the critically injured. Injury 1995; 26: 177-180.

16. Erdil A, Saka M, Ates Y, Tuzun A, Bagci S, Uygun Al. Enteral Nutrition vía percutaneous endoscopic gastrostomy. J Gastroenterrol Hepatol. 2005; 20: 1002-1007.

17. Gauderer M, Ponsky J, Izant R. Gastrostomy without laparotomy a percutaneous endoscopic technique. J Pediatr Surg. 1980; 15:872875.

18. McClave S, Marsano L. Enteral access for nutrition support. J Clin Gastroenterol 2002; 35: 209-213.

19. MinardG, Kudsk D. Is early feeding beneficial? How early is early?. New Horiz 1994; 2:156-163.

20. Mackenzie S., Zygun D., Whitomore L. Implementation of nutritional support protocol JPEN 2005; 29: 74-80.

21. Heyland DK, Dhaliwal R, Drover JW, Gramlich L, Dodek P. Canadian clinical practice guidelines for nutrition support in mechanical ventilated critically ill adult patients. JPEN 2003; 27: 335-375

22. Zaloga G.P., Roberts P., Merik Kp. Feeding the hemodynamically unstable patient a critical evaluation of the evidence. Nut Clin Pract 2003; 18: 285-293.

23. Sánchez M.A. Abdomen abierto. Acta Med Costarric. 2000; 42: 7680.

24. Lee J, Benjamín D, Rendón D. Nutrition support strategies for severely burned patients. Nut Clin Pract 2005; 20: 325-330.

25. Hirshberg A., Walden R. Damage control for abdominal trauma. Surg Clin North Am 1997; 77: 813-820.

26. Fernández L, Norwood S, Roettger R, Wilkins H. Temporary intravenous bag silo closure in severe abdominal trauma. J Trauma 1996; 40: $258-266$.

27. Tsuei. B, Magnuson B. Early nutrition in patients with an open peritoneal cavity. Nut Clin Pract 2003; 18: 253-258.

28. Lara T, Jacob D. Effect of critical illness and nutritional support on mucosal mass and function. Clin Nut 1998; 17: 99-105.

29. Wilmore DW. Catabolic illness. Strategies for enhancing recovery. N Engl J Med. 1991; 325: 695-702.
30. Russell MK, Charney P. Is there a role for specialized enteral nutrition in the intensive care unit?. Nut Clin Pract 2002; 127 : 156-168.

31. Border JR, Chenier R, McManamy RH, La Duca J, Seibel R, Birkhahn R. Multiple system organ failure: muscle fuel deficit with visceral protein malnutrition. Surg Clin North Am 1976; 56: 11471167.

32. Lin MT, Saito H, Fukushima R, Inaba T, Fukatsu K, Inoue T. Route of nutritional supply influences local systemic and remote organ responses to intraperitoneal bacterial challenge. Ann Surg 1996; 223: 84-93

33. Fukatsu K, Kudsk KA, Zarzaur BL, Wu Y, Hanna MK, DeWitt RC. TPN decreases IL-4 and IL-10 mRNA expression in lipopolysaccharide stimulated intestinal lamina propia cells but glutamine preserves the expression. Schock 2001; 15:318-322.

34. Gianotti L, Nelson JL, Alexander JW, Chalk CL, Pyles Tl. Postinjury hypermetabolic response and magnitude of bacterial translocation: prevention with early enteral nutrition. Nutrition 1994; 10:225-231.

35. Ruthig. D.J., Meckling-Gill K.A.. n-3 and n-6 fatty acids stimulate restitution by independent mechanisms in the IEC-6 model of intestinal wound healing. J Nutr Biochem. 2002; 13:27-35.

36. Fell J, Paintin M, Arnaud F. Mucosal healing and a fall in mucosal por-inflamatory cytokine mRNA induce by a specific oral polymeric diet in pediatric Chron's disease. Aliment Pharmacol. Ther 2000; 14: 281-189.

37. Zaloga G.P. Immune-enhancing diets. Where is the beef?. Crit Care Med 1998; 26: 1143-1146.

38. Kudsk K, Moore. Proceedings from summit of I.E.E.T. JPEN 2001; 25: S1-S63.

39. Jeejeebhoy K.N. Enteral Feeding. Curr Opin. Gastroenterol. 2005; 21:187-191.

40. Heyland D.K. Immunonutrition in the critically ill patient. Nutr Clin Pract 2002; 17: 267-272.

41. Charney P. Enteral nutrition: indications, options and formulas. ASPEN. Kendall Hunt; Dubuque I.A., 2001: 145.

42. Mendez C, Craig J, Marsano L. Albumin therapy in clinical practice. Nut Clin Pract 2005; 20:314.

43. Wooley J. Pmerantz R. The efficacy of an enteral access protocol for feeding trauma . Nut Clin Pract 2005; 20:348-353.

44. Peng Y Z., Yuan Z. Effects of early enteral feeding. Burns 2001; 27:145-149.

45. Cerra FB, McPherson JP, Konstantinides FN, Konstantinides NN, Teasley KM. Enteral Nutrition does not prevent M.OF. after sepsis. Surgery 1988; 104: 727-733.

46. Arguello M., Nutrición enteral temprana en paciente críticamente enfermo. Acta Med Costarric 2001; 43 (supl 1): 15 (trabajos libres).

47. Sánchez M.A.. Nutrición pos-operatoria inmediata. Lecturas sobre nutrición 2002; 9 : 42-47.

48. Boullata J. Enteral nutrition in the critically ill. Nutr Clin Pract 2002; 17:137-139.

49. Mc Clave SA, Chang WK. Feeding the hypotensive patient. Ischemic bowel. Nutr Clin Pract 2003; 18: 279-284.

50. Malangoni M.A. Pathogenesis and treatment of intraabdominal infection. Surg Gynecol Obstet. 1990; 171: 3134.

51. Kompan R, Kremzar B, Godzijer E, Prosek M. Effects of early enteral nutrition on intestinal permeability and the development of multi organ failure after multiple injury. Intensive Care Med. 1999; 25:157161.

52. Rapp RP, Young B, Twyman D, Bivins BA, Haack D, Tibbs PA. The favorable effect of early parenteral feeding survival in head-injured patients. J Neurosurg. 1983; 58:906-912. 